research article

\title{
Management of cutaneous side effects of cetuximab therapy with vitamin $\mathrm{K} 1$ crème
}

\author{
Janja Ocvirk and Martina Rebersek \\ Department of Medical Oncology, Institute of Oncology Ljubljana, Ljubljana, Slovenia
}

\begin{abstract}
Background. Cetuximab is a chimeric human-murine monoclonal antibody against the epidermal growth factor receptor (EGFR). It has shown activities against multiple malignancies in clinical trials. EGFR-inhibitors often cause skin toxicity, most frequently acneiform eruption. Xerosis, eczema, fissures, teleangiectasias, nail changes and paronychia can be seen in some cases, rarely hyperpigmentation. The management of the skin toxicity helps patients to overcome cetuximab-associated skin toxicity and is of great importance for patients' compliance. It is generally manageable with standard topical or systemic antibiotics and antiinflammatory agents. The education of patients prior to beginning the therapy and proactive intervention at the first signs of skin toxicity are keys to the successful management. The aim of our study was to investigate cutaneous side-effects of the treatment with cetuximab and to determine the efficacy of vitamin K1 crème.
\end{abstract}

Methods. From September 2006 to August 200730 patients with metastatic colorectal cancer were treated with cetuximab in combination with chemotherapy and suffered from acne-like rash. They were followed at least 3 months, once per week. Skin care was taken with creme with urea and $0.1 \% \mathrm{~K} 1$ vitamin (Reconval $\mathrm{K}{ }^{\circledR}$ ) topically starting after first documented cutaneous toxicity, and was evaluated according to NCI CTCAE, ver.3.

Results. Of 30 patients 6 had grade 3 rash, 18 patients grade 2 and 6 patients grade 1 . Reconval K1 ${ }^{\circledR}$ was used twice daily. In all patients we observed the improvement of cutaneous toxicity. The median improvement was 8 days and 18 days to observe down-staging in rash at least for 1 grade. In only 3 of 6 patients with grade 3 toxicity the reduction of cetuximab dose was needed. In historical controls in all patients with grade 3 the reduction of cetuximab dose was recommended and performed. No dose reduction or delay of treatment was needed in group of patients with grade 1 and 2 cutaneous toxicity. We didn't observe any local or systemic toxicity of topical use of Reconval $\mathrm{K} 1^{\circledR}$.

Conclusions. To our knowledge this is the first documented effect of topical use of K1 vitamin crème for reducing cetuximab induced cutaneous toxicity in patients with metastatic colorectal cancer. We conclude that Reconval $\mathrm{K} 1^{\circledR}$ is useful in skin care in patients treated with cetuximab. Further studies are needed to evaluate the impact on response rate of cetuximab and quality of life.

Key words: cetuximab; cutaneous side-effects; vitamin K1 crème; colorectal cancer

Received 26 November 2008

Accepted 5 December 2008

Correspondence to: Janja Ocvirk, MD, PhD, Institute of Oncology Ljubljana, Zaloska 2, Ljubljana. Phone: +386 15879 220; Fax: +386 15879 305; E-mail: jocvirk@onko-i.si 


\section{Introduction}

Cetuximab is a chimeric immunoglobulin G1 (IgG1) monoclonal antibody that binds to the extracellular domain of epidermal growth factor receptor (EGFR) with high specificity and a higher affinity than that of epidermal growth factors, thus blocking ligand-induced phosphorylation of EGFR. The affinity is approximately 5 to 10 -fold higher than that of endogenous ligands. ${ }^{1,2}$

EGFR (c-erbB1 or HER1) is a member of the ErbB family of tyrosine kinase receptors. EGFR is a 170-kd cell surface protein composed of 3 regions: an extracellular ligand-binding domain, a hydrophobic transmembrane domain, and an intracellular domain with adenosine triphosphate (ATP)-dependent tyrosine kinase activity. ${ }^{3}$ EGFR signalling pathways are involved in the control of cell survival, cell cycle progression, angiogenesis, cell migration and cellular invasion/metastasis. ${ }^{4}$

EGFR is found to be overexpressed in many of human tumours: colorectal, gastric, oesophageal, head and neck, lung, breast and prostate cancer, as well as glioblastoma, bladder and ovarian carcinoma. ${ }^{2,5}$

Binding of the antibody to EGFR prevents the stimulation of the receptor by endogenous ligands and results in inhibition of cell proliferation, enhanced apoptosis, and reduced angiogenesis, invasiveness and metastasizing. Binding of cetuximab to the receptor also results in internalization of the antibody-receptor complex which leads to an overall downregulation of EGFR expression. ${ }^{6}$ Preclinical studies have demonstrated that cetuximab reduces the resistance to chemotherapy and radiotherapy of human tumour cell lines in vitro and of nude mice bearing xenografts of human tumours. In clinical and preclinical studies, cetuximab has been shown to induce a response to treatment when used in combination with chemotherapy in the patients previously refractory to chemotherapy. ${ }^{6}$

\section{Clinical efficacy of cetuximab}

Cetuximab in combination with irinotecan and oxaliplatin is approved for the treatment of patients with epidermal growth factor receptor (EGFR)-expression in metastatic colorectal cancer. In combination with radiation therapy, it is indicated for the treatment of patients with locally advanced squamous cell cancer of the head and neck (SCCHN). ${ }^{4}$

\section{Metastatic colorectal cancer (CRC)}

The approval of cetuximab followed the positive results of BOND trial; the combination of cetuximab plus irinotecan induced a response rate of $23 \%$ in the patients with irinotecan refractory EGFR-positive metastatic CRC and tumour stabilization in 33\% of patients. The response rate after cetuximab alone in this trial was $11 \%{ }^{7}$

The data from the large study (MABEL) confirmed the activity of cetuximab in heavily pre-treated patients. The overall response rate was $20 \%$, the disease control rate was $45 \%$ and the survival estimate was at that time 9.2 months. $^{8}$

The EPIC study investigated the overall survival of the second-line irinotecan versus irinotecan plus cetuximab in metastatic CRC patients after the progression or intolerance of the first-line oxaliplatin based regimen. The addition of cetuximab to irinotecan led to a significant increase in RR (16.4 vs 4.2), a significant increase in disease control (61.4 vs 45.8), and a 30\% reduction in the risk of disease progression. ${ }^{9}$

Cetuximab was used in the first-line therapy in several small trials and showed promising results. $^{2}$ There are also trials showing the effectiveness of cetuximab in 
Table 1. Simplified classification of acneiform eruption caused by EGFR inhibitors

\begin{tabular}{llll}
\hline A/ NCI CTC v 3.0 & & & \\
\hline Rash/desquamation & & & \\
$\begin{array}{l}\text { Gradus 1 } \\
\text { Lesions without } \\
\text { symptoms }\end{array}$ & Gradus 2 & $\begin{array}{l}\text { Gradus 3 } \\
\text { Lesions with symptoms }\end{array}$ & Lesions with symptoms \\
& $<50 \%$ body surface & $\begin{array}{l}\text { Exfoliative or ulcerative } \\
\text { erythroderma }\end{array}$ \\
\hline
\end{tabular}

B/ NCI CTCAE v3.0

Rash/desquamation

Gradus 1

Gradus 2

Gradus 3

Gradus 4

Lesions without

Lesions with symptoms Lesions with symptoms

Exfoliative ulcerative or

symptoms $\geq 50 \%$ body surface

bullous erythroderma

$<50 \%$ body surface

Rash: acne/acneiform

\begin{tabular}{llll}
\hline Gradus 1 & Gradus 2 & Gradus 3 & Gradus 4 \\
$\begin{array}{l}\text { Intervention not } \\
\text { indicated }\end{array}$ & $\begin{array}{l}\text { Intervention not } \\
\text { indicated }\end{array}$ & $\begin{array}{l}\text { Pain, disfigurement, } \\
\text { ulceration or } \\
\text { desquamation }\end{array}$ & - \\
\hline
\end{tabular}

the first-line treatment of mCRC: a phase III study (CRYSTAL trial) comparing standard FOLFIRI alone with cetuximab in combination with FOLFIRI proved the effectiveness of cetuximab. With the use of cetuximab, the median progression-free survival was significantly longer (8.9 months vs 8 month), and the response rate increased (46.9\% vs $38.7 \%)$, thereby reducing the relative risk of progression by approximately $15 \%{ }^{10}$

The large phase II study comparing cetuximab plus FOLFOX-4 with FOLFOX-4 alone in the first-line treatment (OPUS trial) showed that the combination was effective and safe. The overall response rate increased by $10 \%{ }^{11}$

Cetuximab is also effective in combination with the angiogenesis inhibitor bevacizumab. The phase II trial (BOND-2 study) showed that the combination therapy with cetuximab, bevacizumab and irinotecan, compared to cetuximab and bevacizumab alone, improved the efficacy. After irinotecan failure, this combination increased the response rate and prolonged the time to progression (response rate was $37 \%$ vs $23 \%$ and median time to progression 7.9 months vs 5.6 months). ${ }^{12}$

The combination therapy with cetuximab after the failure of the conventional therapy increases resectability rates without increasing operative mortality or liver injury. Adam and colleagues showed that $7 \%$ of patients treated with cetuximab after the failure of the conventional therapy experienced a treatment response that allowed curative hepatectomy. These patients were unresectable after two or more lines of conventional treatment and prior to the initiation of the treatment with cetuximab. ${ }^{13}$

The results from phase I study ${ }^{14}$ and phase II study showed that cetuximab could be safely administered every second week. $^{15}$ 
Squamous cell carcinoma of the head and neck (SCCHN)

Cetuximab plus radiotherapy show a significant efficacy benefit over radiotherapy alone in the treatment of locally advanced SCCHN. The risk of the locoregional progression was $32 \%$ lower with the use of cetuximab plus radiotherapy than with radiotherapy alone. Cetuximab plus radiotherapy also demonstrated a significant improvement in the median overall survival versus radiotherapy alone. Also the risk of death was $26 \%$ lower with the use of cetuximab plus radiotherapy compared to radiotherapy alone. ${ }^{16}$

Cetuximab was investigated in the second-line and first-line settings for the patients with recurrent/metastatic SCCHN following the failure of surgery or radiotherapy. In the phase III randomized comparison of cisplatin plus cetuximab or cisplatin plus placebo, the addition of cetuximab to cisplatin increased the response rate to $26 \%$ and $10 \%$, respectively, and was also associated with the trend towards a prolonged median overall survival (9.2 vs 8.0 months). ${ }^{17}$

The phase I study showed that the addition of cetuximab to a platinum/5-FU combination in the treatment of patients with recurrent and/or metastatic SCCHN was active and well tolerated in the first-line settings. 18

Cetuximab was also effective in nasopharyngeal carcinoma: the combination of cetuximab plus carboplatin showed an important clinical benefit in the patients with recurrent and/or metastatic nasopharyngeal carcinoma failing chemotherapy with an overall response rate of $12 \%$ and median overall survival of 7.6 months. ${ }^{19}$

\section{Other indications}

In the phase I and II studies, it was shown that the combination of capecitabine plus cetuximab together with radiotherapy, as well as capecitabine, oxaliplatine plus cetuximab together with radiotherapy could be feasible and safe regimens for rectal cancer. $^{2}$

Other phase II and III studies showed a significant response to treatment in variable proportions of patients with non-small cell lung cancer (NSCLC) when cetuximab was used as the first- or second-line in combination with chemotherapy. ${ }^{6}$

\section{Safety profile and side effects}

Cetuximab is well-tolerated in cancer patients both as a single agent and in combination with other anti-cancer agents or radiation. Skin reactions may develop in more than $80 \%$ of patients and mainly present as acne-like rash and/or, less frequently, as pruritus, dry skin, desquamation, hypertrichosis, or nail disorders (e.g. paronychia). Approximately $15 \%$ of the skin reactions are severe, including single cases of skin necrosis. The majority of skin reactions develop within the first three weeks of therapy. They generally resolve, without sequelae, over the course of the following weeks and months even when the treatment is continued. $2,7,20$

In combination with irinotecan, additional reported undesirable effects were those related to irinotecan (such as diarrhoea, nausea, vomiting, mucositis, stomatitis, fever, leukopenia, alopecia).

In combination with the local radiation therapy of the head and neck area, additional undesirable effects were those typical of the radiation therapy (such as mucositis, radiation dermatitis, dysphagia or leucopoenia, mainly presenting as lymphocytopenia). ${ }^{4}$

Anaphylactic hypersensitivity reactions, characterized by a rapid onset of airway obstruction, urticaria, and hypotension, occurred in 3\% of patients. Approximately 
$90 \%$ of the reactions developed with the first cetuximab infusion, despite premedication with antihistamines.

Other serious adverse events during cetuximab monotherapy, with the incidence of $5 \%$ or less, included interstitial lung disease, fever, sepsis, kidney failure, pulmonary embolus, dehydration, and diarrhoea. ${ }^{4}$ Progressively decreasing serum magnesium levels which, in some patients, developed into severe hypomagnesaemia, were also noted. ${ }^{21}$

The EGFR is important for the normal skin development and the function. ${ }^{22}$ In normal adult human epidermis, EGFR is strongly expressed in keratinocytes, in the sebaceous glands, and also in the epithelium of hair follicles. The expression is higher in the basal layer of the epidermis and in the outer root sheath of hair follicles, where the keratinocytes are proliferating and are undifferentiated. After the therapy with EGFR inhibitor, histopathological findings of the skin showed a thinner and more compact stratum corneum as well as prominent keratin plugs. ${ }^{23}$ Thus, blocking the EGFR pathway leads to a unique group of skin reactions dominated by an acne-like eruptions, eczema, xerosis, changes of the hair and nails.

Vitamin $\mathrm{K}$ is activator EGFR pathway. The use of vitamin $\mathrm{K}$ crème can reduce skin toxicity caused by EGFR inhibitors. ${ }^{24}$ The aim of our study was to determine the efficacy of vitamin $\mathrm{K} 1$ crème.

\section{Patients and methods}

The treatment of metastatic colorectal cancer with cetuximab after the failure of the treatment with irinotecan was first performed in Slovenia in 2005. During the period from September 2006 to August 2007, thirty patients with metastatic colorectal cancer were treated with cetuximab in com- bination with chemotherapy and suffered from acne-like rash.

The patients with the typical acneiform rash skin reactions were included. They occurred from the sixth day after the first application of cetuximab to 14 days at the latest. They were followed once per week at least 3 months. For the clinical improvement was relevant the improvement of skin adverse reactions at each control. Skin care was taken with crème with urea and $0.1 \%$ $\mathrm{K} 1$ vitamin (Reconval $\mathrm{K} 1{ }^{\circledR}$ ) topically twice per day starting after the first documented cutaneous toxicity, It was classified according to the National Cancer Institute Common Toxicity Criteria, Version 3.0 (NCI CTC v 3.0 (Table 1). ${ }^{25}$

Erbitux was administered at a loading dose of $400 \mathrm{mg} / \mathrm{m}^{2}$ infused over 2 hours, followed by a weekly dose of $250 \mathrm{mg} / \mathrm{m}^{2}$ infused over 1 hour. The patients were premedicated with a histamine ${ }_{1}$ antagonist with or without corticosteroids.

\section{Results}

The treatment with cetuximab showed a great efficacy with the evident skin toxicity. The most common side effect seen with cetuximab was an acneiform skin rash. The following skin reactions were also observed: hair and nail changes, rhagades, paronychia, trichomegalia, and uveitis.

\section{Acneiform rash skin reactions}

Acneiform eruptions were more or less confined to the seborrhoea areas which are rich in sebaceous glands (face, neck, scalp, shoulders, upper trunk and chest in Vshaped patterns) (Figures 1-3). Occasionally, they affected lower parts of the back and abdomen, but rarely arms and legs.

Six out of 30 patients experienced grade 3,18 patients grade 2 , and 6 patients grade 


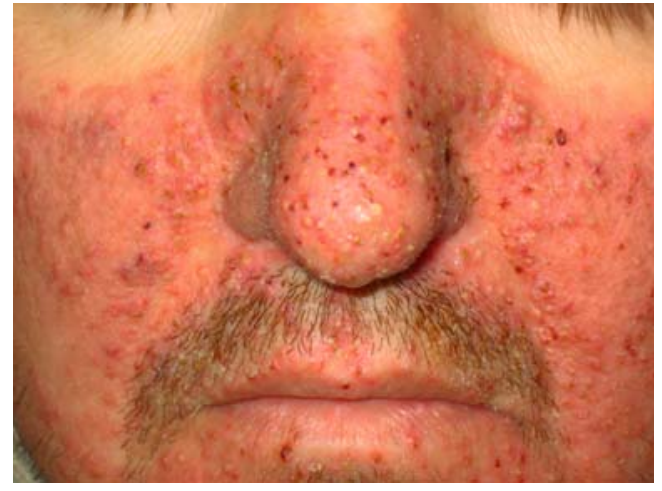

Figure 1. Tipical acneiform eruption caused by EGFR inhibitors.

1 acneiform rash skin reaction at the time we started to manage skin rash.

Acneiform eruptions compared to the acne vulgaris are different and are appearing on the changed skin which is very dry and

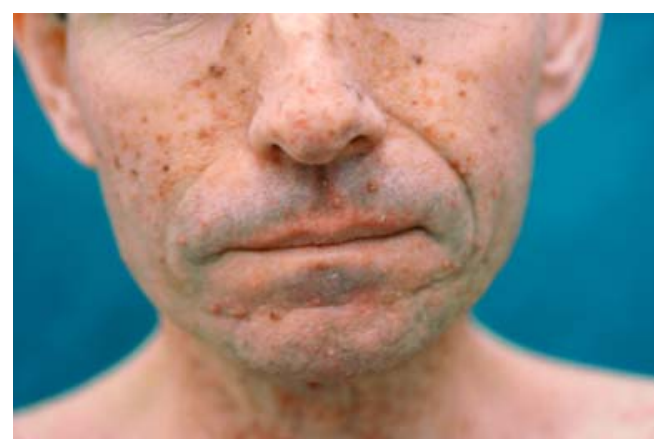

Figure 3. Tipical acneiform eruption caused by EGFR inhibitors.

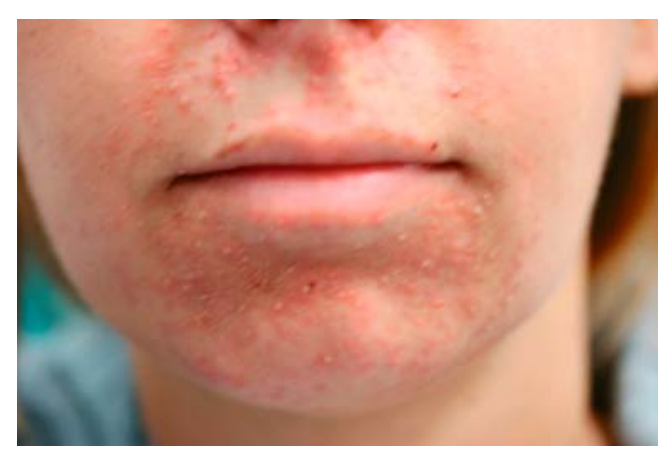

Figure 4a. Acneiform rash before treatment with vitamin K1 crème.

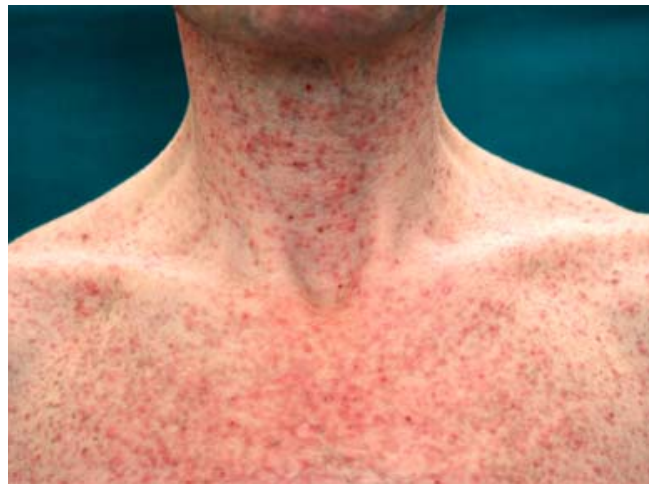

Figure 2. Tipical acneiform eruption caused by EGFR inhibitors.

disposed to cracks. The skin lesions consist of follicular papules, which may evolve into pustules and possibly dry out with the formation of yellow crusts. Skin lesions can be accompanied by pruritus and xerosis. The aetiology of acneiform eruptions differs from that of acne vulgaris; so, the treatment generally used for acne vulgaris is not helpful and we do not advise it (i.e. the treatment with benzoyl peroxide).

In all patients skin care with $\mathrm{K} 1$ crème twice per day was performed. For grade 2 acneiform eruptions, topical antibiotic preparations, mostly clindamycin (1\%) and erythromycin, are used according to the antibiograms and experiences, concomitantly with vitamin $\mathrm{K} 1$ crème, when pustules were observed. We used systemic antibiotics in 4

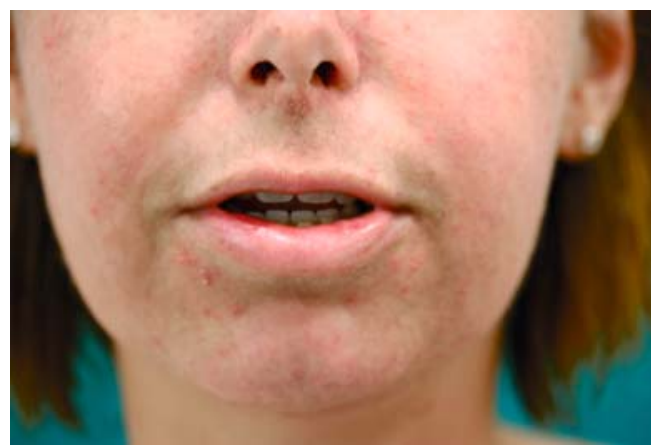

Figure $4 b$. The scin after 1 week of using vitamin K1 crème. 


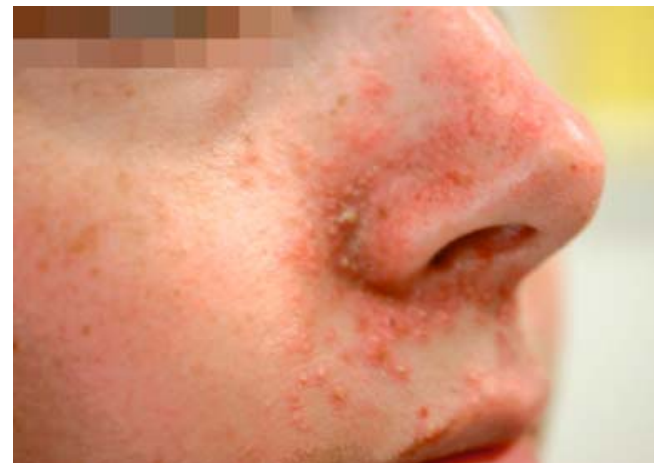

Figure 5a. Acneiform rash before treatment with vitamin $\mathrm{K} 1$ crème.

patients with G3 skin rash and topical antibiotics in 2 patients with G3 and 5 patients with G2 skin rash.

Median observed time for the improvement of skin toxicity was 8 days, but according to patients' report it was even shorter. We also recorded lowering of grade of skin rash. Median time for the improvement of skin rash of all grades was 18 days.

The results of the improvement of skin rash are presented in figures (Figures 4-7).

In case of grade 3 reaction, the treatment with cetuximab should be discontinued until the reactions resolve recording to $\mathrm{SmPC}$ of drug. The treatment can be restarted at a lower dose. We discontinued the treatment

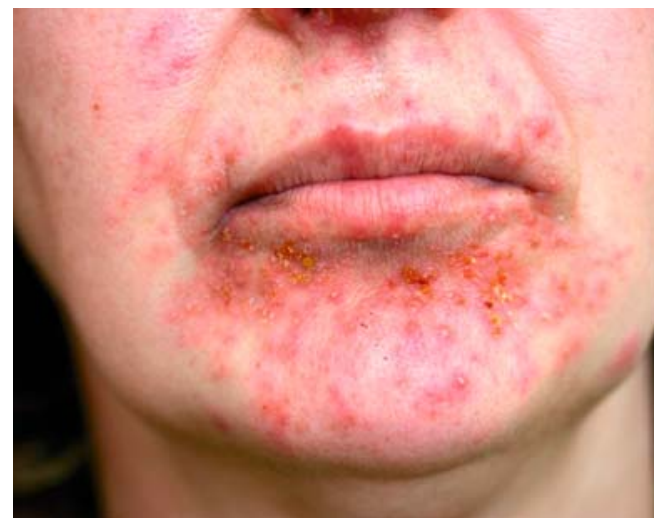

Figure 6a. Acneiform rash before treatment with vitamin $\mathrm{K} 1$ crème.

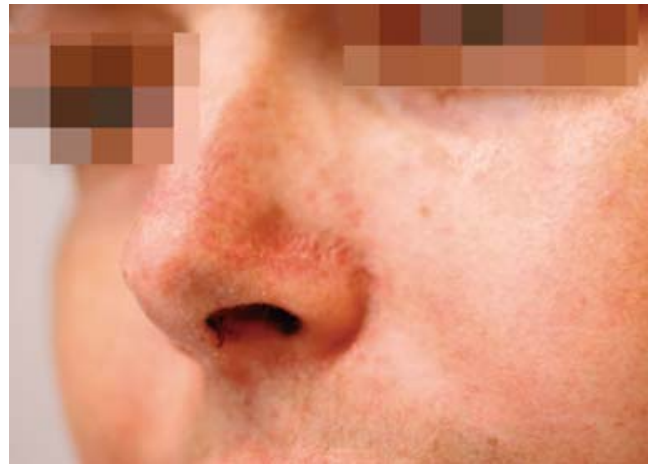

Figure $\mathbf{5 b}$. The scin after 1 week of using vitamin K1 crème.

in only 3 patients with G3 skin rash; no case of grade 4 skin rash was reported.

Other less common reactions are nail changes (paronychia), hair modifications, xeroses trichomegalia, dry itchy skin, rhagades, uveitis and conjunctivitis, telangiectasias, hyperpigmentation, fissures in genital and anal region.

In a group of patients, where we used vitamin K1 crème, 3 and 6 months after the treatment we did not observe any xeroderma or telangiectasias, even they are frequently noticed in the cetuximab treatment; especially we observed them in patients before using vitamin $\mathrm{K} 1$ crème.

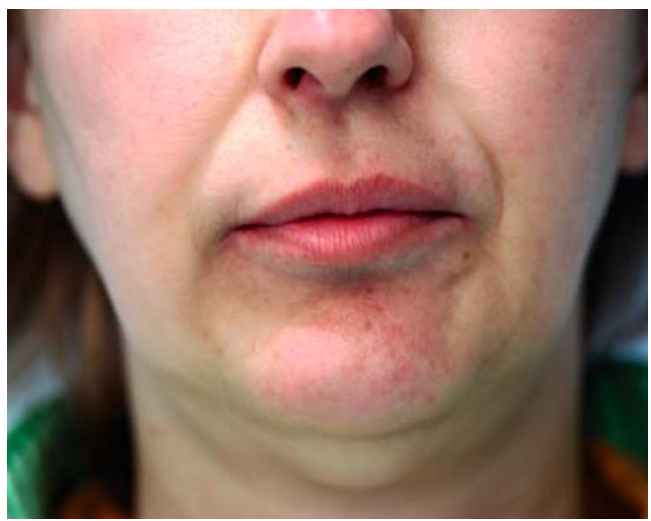

Figure $\mathbf{6 b}$. The skin after 1 week of using vitamin K1 crème. 


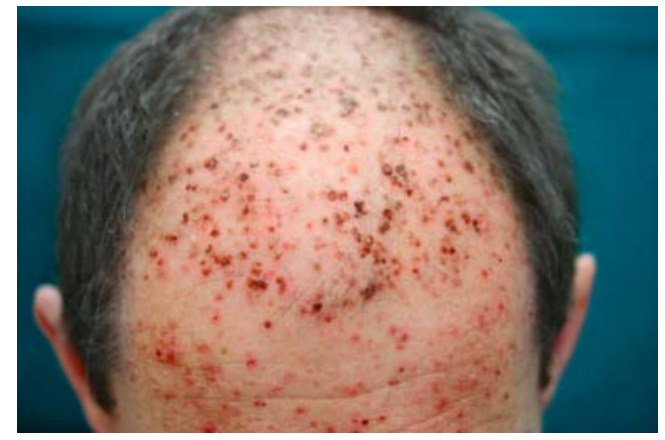

Figure 7a. Acneiform rash before treatment with vitamin $\mathrm{K} 1$ crème.

\section{Discussion}

Cetuximab is a monoclonal antibody which is registered for the treatment of metastatic colorectal cancer, head and neck cancers and lately also in the treatment of NSCLC. By blocking EGFR inhibits the proliferation of different types of tumour cells, as well as normal cells expressing EGFR, which are in skin, intestinal mucus and liver cells. The overexpression of EGFR in the epidermis, sweat glands, hair follicles and endotelium cells is reflected as an adverse reaction on the skin, mucus membranes, hair and nails. ${ }^{20,26}$

Skin toxicities are especially acneiform eruption, xerosis, fissures of palms and foot, paronychia and changes in hair growth. Aetiology and signs of skin reactions of patients in our study were very similar as described in many articles. ${ }^{27-29}$

Adverse events are related to the dose and are more intensive at high dose; by the lowering of the dose, the intensity of adverse events decreases, too. The correlation between the extent and/or severity of the acneiform eruption and anti-tumour efficacy of cetuximab, which was reported in literature, was observed also in our patients. ${ }^{20}$

Therapeutic methods for the management of skin adverse effects observed in the

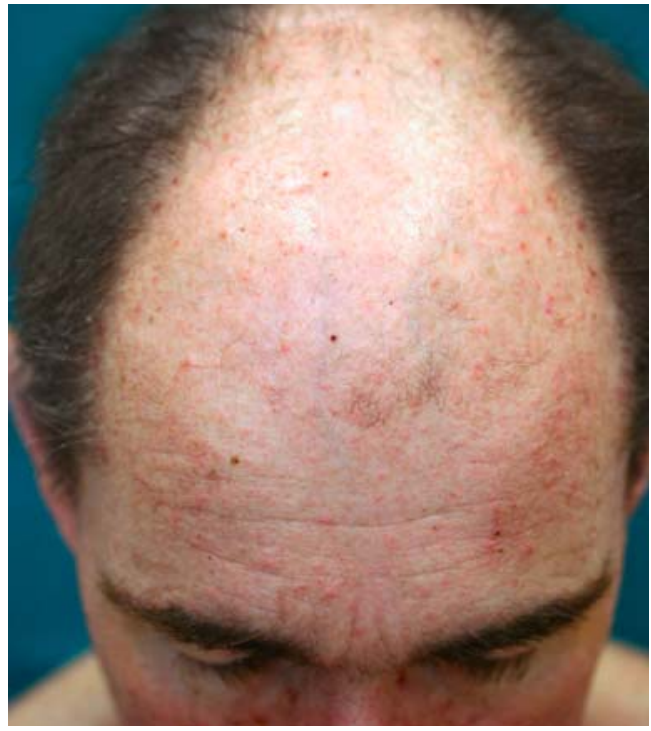

Figure $\mathbf{7 b}$. The skin after 1 week of using vitamin K1 crème.

treatment with cetuximab are not standardized because there are no effective products to treat them and, above all, because published data are very contradictory.

In all patients with G1 acneiform rash we used only vitamin $\mathrm{K} 1$ crème. In patients with super-infections of skin presented as pustules, we used also topic or systemic antibiotics according to clinical references and the extent of infection (G2 and G3 side effects). We also used $1 \%$ topic antibiotic clindamicyn in 5 of 18 patients with G2 skin toxicity, namely, pustules and 2 with G3 skin toxicity. We often decided for the use of systemic antibiotic in such cases according to previous experiences. According to our experiences, we advise not to use benzoyl peroxide for the management of acneiform eruptions, as well as not to use corticosteriods.

For the patients who have grade 1 acneiform eruptions, the treatment with vitamin $\mathrm{K} 1$ crème alone was enough to manage skin toxicity. For the patients with grade 2 skin eruptions, the addition of topical antibiotics to the already mentioned therapy is recom- 
mended for those with pustules; especially clindamycin is clinically proved and according to its antibiogram considered to be the most effective antibiotic. The appropriate treatment of the patients with grade 3 skin reactions is systemic treatment with antibiotic clindamycin and topically with vitamin $\mathrm{K} 1$ crème.

\section{Conclusions}

It is very important to recognize timely and accurately the skin adverse effects of EGFR inhibitors and to treat them promptly in order to assure a better quality of life to the patients during the treatment. In addition to traditional endpoints, such as disease control and survival, the quality of life became more and more important. ${ }^{27,30}$ The proper management of skin reactions enables the longer treatment with cetuximab, without the dose reduction or drug discontinuation. The continuous treatment assures effective treatment, better response to treatment, and longer survival of patients, which is the most important goal to be achieved.

Patients have to be encouraged to follow physician's advice regarding the treatment of dermatological side effects. The appropriate treatment of side effects allows the patients to continue receiving the therapy without dose reduction or drug discontinuation and to have better outcome of the treatment.

The study performed at our clinic is the first documented effect of topical use of K1 vitamin crème for reducing cetuximab induced cutaneous toxicity in patients with metastatic colorectal cancer. We conclude that Reconval $K 1^{\circledR}$ is useful in skin care in patients treated with cetuximab. Further studies are needed to evaluate the impact on the response rate of cetuximab and quality of life.

\section{References}

1. Baselga J. Why the epidermal growth factor receptor? The rationale for cancer therapy. Oncologist 2002; 7(Suppl 4): 2-8

2. Moosmann N, Heinemann V. Cetuximab in the treatment of metastatic colorectal cancer. Expert Opin Biol Ther 2007; 7: 243-56.

3. Frieze DA, McCune JS. Current status of cetuximab for the treatment of patients with solid tumors. Ann Pharmacother 2006; 40: 241-50.

4. Summary of product characteristics. http://www. emea.europa.eu/humandocs/Humans/EPAR/erbitux/erbitux.htm; 25 November 2008.

5. Smrdel U, Kovač V. Erlotinib in previously treated non-small-cell lung cancer. Radiol Oncol 2006; 40: $39-42$.

6. Harding J, Burtness B. Cetuximab: an epidermal growth factor receptor chimeric human-murine monoclonal antibody. Drugs Today 2005; 41: 107127.

7. Cunningham D, Humblet $Y$, Siena S, Khayat D, Bleiberg H, Santoro A, et al. Cetuximab monotherapy and cetuximab plus irinotecan in irinotecan-refractory metastatic colorectal cancer. $N$ Eng J Med 2004; 351: 337-45.

8. Wilke H, Glynne-Jones R, Thaler J, Adenis A, Preusser P, Aguilar EA, et al. Cetuximab plus irinotecan in heavily pretreated metastatic colorectal cancer progressing on irinotecan: MABEL Study. J Clin Oncol 2008; 26: 5335-43.

9. Scheiuthauer W, Sobrero A, Lenz HJ, Maurel J, Lutz M, Middleton G, et al. Cetuximab plus irinotecan in patients with mCRC who failed prior oxaliplatin-based therapy: the EPIC trial. [Abstract]. Eur J Cancer 2007; 5(4): 235.

10. Van Cutsem E, Nowacki M, Lang I, Cascinu S, Schepotin I, Maurel J, et al. Randomized phase III study of irinotecan and 5-FU/FA with or without cetuximab in the first-line treatment of patients with metastatic colorectal cancer $(\mathrm{mCRC})$ : the CRYSTAL trial. ASCO 2007. J Clin Oncol 2007; 25(18 Suppl): Abstract 4000.

11. Bokemeyer C, Staroslawska E, Makhson A, Bondarenko I, Hartmann JT, Shelygin Y, et al. Cetuximab plus 5-FU/FA/oxaliplatin (FOLFOX-4) versus FOLFOX-4 in the first-line treatment of metastatic colorectal cancer (mCRC): a large-scale phase II study, OPUS. [Abstract]. Eur J Cancer 2007; 5(4): 236. 
12. Salz LB, Lenz HJ, Kindler HL, Hochster HS, Wadler S, Hoff PM, et al. Randomized phase II trial of cetuximab, bevacizumab, and irinotecan compared with cetuximab and bevacizumab alone in irinotecan-refractory colorectal cancer. The BOND-2 study. J Clin Oncol 2007; 25: 4516-8.

13. Adam R, Aloia T, Levi F, de Haas RJ, Paule B, Bralet MP, et al. Hepatic resection after rescue cetuximab treatment for colorectal liver metastases previously refractory to conventional systemic treatment. J Clin Oncol 2007; 25: 4593-602.

14. Ciardiello F, Cervantes A, Vega-Villegas ME, Casado E, Rodriguez-Braun E, Martinelli E, et al. Optimal dose for an every 2 week (q2w) cetuximab (C) regimen in patients (pts) metastatic colorectal cancer (mCRC): a phase I safety, pharmacokinetics(PK) and pharmacodynamics (PD) study of weekly (q1w) add q2w schedules. [Abstract]. Eur J Cancer 2007; 5(4): 247.

15. Pfeiffer P, Bjerregaard JK, Qvortrup C, Jensen BV, Yilmaz M, Nielsen D. Simplification of cetuximab (Cet) administration: double dose of every second week as a 60 minute infusion. J Clin Oncol 2007; 25(18 Suppl): Abstract 4133.

16. Bonner JA, Harari PM, Giralt J, Azarnia N, Shin $\mathrm{DM}$, Cohen RB, et al. Radiotherapy plus cetuximab for squamous-cell carcinoma of the head and neck. N Eng J Med 2006; 354: 567-78.

17. Burtness B, Goldwasser MA, Flood W, Mattar B, Forrastiere AA. Phase III randomized trial of cisplatin plus cetuximab in metastatic/recurrent head and neck cancer: An eastern Cooperative Oncology Group study. J Clin Oncol 2005; 23: 8646-54.

18. Bourhis J, Rivera F, Mesia R, Awada A, Geoffrois L, Borel C, et al. Phase I/II study of cetuximab in combination with cisplatin or carboplatin and fluorouracil in patients with recurrent or metastatic squamous cell carcinoma of the head and neck. J Clin Oncol 2006; 24: 2866-72.

19. Bernier J. Cetuximab in the treatment of head and neck cancer. Expert Rev Anticancer Ther 2006; 6: 1539-52.

20. Saltz LB, Meropol NJ, Loehrer PJ Sr, Needle MN, Kopit J, Mayer RJ. Phase II trial of cetuximab in patients with refractory colorectal cancer that expresses the epidermal growth factor receptor. $J$ Clin Oncol 2004; 22: 1201-8.

21. Carson EJ, Novak AM, Stella PJ. Hypomagnesemia in patients with stage IV colorectal cancer treated with cetuximab as a single agent. [Abstract 3655]. Proc Am Soc Clin Oncol 2005; 24: 248-54.
22. Vallbohmer D, Lenz HJ. Epidermal growth factor receptor as a target for chemotherapy. Clin Colorectal Cancer 2005; 5(Suppl 1): S19-27.

23. Damjanov N, Meropol NJ. Epidermal growth factor receptor inhibitors for the treatment of colorectal cancer: a promise fulfilled? Oncology 2004; 18: 479-88.

24. Perez- Soler R, Zou Y, Li T, Tornos C, Ling Y. Topical vitamin K3 (Vit K3, Menadione) prevents erlotinib and cetuximab-induced EGFR inhibition in the skin. J Clin Oncol 2006; 24(18 Suppl): Abstract 3036.

25. National Cancer Institute. Common terminology criteria for adverse events v.3.0 http:// ctep.cancer. gov/reporting/ctc.html.

26. Busam KJ, Capodieci P, Motzer R, Kiehn T, Phelan D, Halpern AC. Cutaneous side-effects in cancer patients treated with the epidermal growth factor receptor antibody C225. Br J Dermatol 2001; 144: 1169-76.

27. Segaert1 S, Van Cutsem E. Clinical signs, pathophysiology and management of skin toxicity during therapy with epidermal growth factor receptor inhibitors Ann Oncol 2005; 16: 1425-33.

28. Agero ALC, Dusza SW, Benvenuto-Andrade C, Busam KJ, Myskowski P, Halpern AC. Dermatological side-effects associated with the epidermal growth factor receptor inhibitors. $J \mathrm{Am}$ Acad Dermatol 2006; 55: 657-70.

29. Galimont-Collen AFS, Vos LE, Lavrijsen APM, Ouwrkerk J, Gelderblom H. Classification and management of skin, hair, nail and mucosal sideeffects of epidermal growth factor receptor (EGFR) inhibitors. Eur J Cancer 2007; 43: 845-51.

30. Velenik V, Oblak I, Anderluh F. Quality of life in patients after combined modality treatment of rectal cancer: Report of a prospective phase II study. Radiol Oncol 2008: 42: 207-14. 